\title{
Activation of the ROCK1/MMP-9 pathway is associated with the invasion and poor prognosis in papillary thyroid carcinoma
}

\author{
DINGYUAN LUO ${ }^{1,2^{*}}$, HAIBO CHEN $^{2,3^{*}}$, XIAOJUAN LI ${ }^{2,4^{*}}$, PENGHUI LU $^{1 *}$, MIAOYUN LONG $^{1}$, \\ XINZHI PENG ${ }^{1}$, SHAOJIAN LIN ${ }^{1}$, LANGPING TAN ${ }^{1}$, YUE ZHU ${ }^{1}$, NENGTAI OUYANG ${ }^{5}$ and HONGHAO LI ${ }^{1}$ \\ ${ }^{1}$ Department of Vascular and Thyroid Surgery, ${ }^{2}$ Guangdong Provincial Key Laboratory of Malignant Tumor Epigenetics and \\ Gene Regulation, Medical Research Center, ${ }^{3}$ Department of Nuclear Medicine, ${ }^{4}$ Center of Medical Research, and \\ ${ }^{5}$ Department of Pathology, Sun Yat-sen Memory Hospital, Sun Yat-sen University, Guangzhou, Guangdong 510120, P.R. China
}

Received November 24, 2016; Accepted July 20, 2017

DOI: $10.3892 /$ ijo.2017.4100

\begin{abstract}
Rho-associated protein kinase 1 (ROCK1), a serine/ threonine kinase, has previously been shown to be overexpressed in various types of human malignant tumors and to play an important role in cancer development and progression. Although ROCK1 has gained growing prominence as an important protein kinase in cancer biology, its potential as a predictive biomarker and a therapeutic target in papillary thyroid carcinoma (PTC) remains unknown. In the present study, ROCK1 expression was examined in 356 formalinfixed, paraffin-embedded papillary thyroid carcinoma tissues using immunohistochemistry, and its clinical implications and prognostic significance were analyzed. Our results showed that ROCK1 expression was significantly increased in PTC compared with normal tissues, and was significantly associated with tumor size, lymphatic metastasis, distant organ metastasis, extrathyroid invasion, vascular invasion and tumor, node and metastasis (TNM) stage. Patients with strong ROCK1 expression had lower overall survival, disease-free survival, lymph node recurrence-free survival and distant
\end{abstract}

Correspondence to: Dr Nengtai Ouyang, Department of Pathology, Sun Yat-sen Memory Hospital, Sun Yat-sen University, 107 Yanjiang West Road, Guangzhou, Guangdong 510120, P.R. China

E-mail: ouynt@mail.sysu.edu.cn

Dr Honghao Li, Department of Vascular and Thyroid Surgery, Sun Yat-sen Memorial Hospital, Sun Yat-sen University, 107 Yanjiang West Road, Guangzhou, Guangdong 510120, P.R. China

E-mail: lihonghao158158@163.com

*Contributed equally

Abbreviations: ROCK1, Rho-associated protein kinase 1; MMP-9, matrix metalloproteinase-9; IHC, immunohistochemistry; PTC, papillary thyroid carcinoma; ECM, extracellular matrix; FBS, fetal bovine serum; SI, staining index; OS, overall survival; DFS, diseasefree survival; LNRFS, lymph node recurrence-free survival; DRFS, distant recurrence-free survival

Key words: ROCK1, MMP-9, metastasis, prognosis, papillary thyroid carcinoma recurrence-free survival rates than those with weak expression. Furthermore, overexpression of ROCK1 in papillary thyroid carcinoma cells was found to increase their invasiveness. Silencing ROCK1 by siRNA, however, caused an inhibition of cell invasion. Knockdown of ROCK1 decreased the volume and weight of the xenograft tumors, while overexpression of ROCK1 showed a proliferative tendency with significantly greater tumor volume and weight in vivo. Moreover, the upregulation of ROCK1 increased the expression of MMP-9, and levels of MMP-9 positively correlated with the ROCK1 levels in PTC tissues, implicating that MMP-9 may be involved in the mechanism of ROCK1 in the development and progression of PTC. These data suggest that ROCK1 might be a potential prognostic marker and therapeutic target for the treatment of PTC.

\section{Introduction}

Papillary thyroid carcinoma (PTC) is the most common thyroid carcinoma, accounting for approximately $80 \%$ of all thyroid malignant tumors (1-4). Despite highly curable and presenting a 10 -year survival rate more than $90 \%$, lymph node metastasis, especially in the neck, occurs in $20-50 \%$ of all tumor patients and regional recurrence is found in $5-20 \%$ of patients who have undergone total thyroidectomy $(3,5,6)$. Moreover, PTC is prone to spread through lymphatic ducts, resulting in recurrence, metastases and even death (3). Recurrent PTC mainly refers to local and distant recurrence, including recurrence of primary tumor, lymph node metastases, invasion of the esophagus and trachea, invasion of muscles, nerves and distant metastases. Many factors could affect the recurrence of thyroid cancer, and 20\% PTC patients relapse after treatment and require reoperation (7-9). Consequently, analyzing the molecular characteristics of PTC and exploring new targets for therapy have been major clinical concerns.

Rho-associated protein kinase 1 (ROCK1), a serine/threonine kinase, affects cell invasion by changing the status of the cytoskeleton (10). Recent studies have shown that ROCK1 overexpression was found in a variety of tumors. ROCK1 plays an important role in the regulation of cell morphology, adhesion and motility. Its inhibitors are capable of reducing cancer cell migration, proliferation and invasion (11-13). ROCK1 
overexpression is related to tumor metastasis, and its inhibition was suggested to be a novel approach for treating breast cancer (14). However, the function of ROCK1 in PTC remains elusive.

Tumor recurrence and distant metastasis are two major factors that are responsible for poor survival of cancer patients $(15,16)$. The degradation of the extracellular matrix (ECM) is necessary for the initiation and development of tumor metastasis (17). This process is mainly influenced by the activity of matrix metalloproteinases (MMPs), which are enzymes that degrade structural components of the ECM, molecules of cell-cell and cell-ECM interactions. MMPs can release and activate growth factors and cytokines, facilitating tumor invasion and the metastatic processes, with many human tumors characterized by increased concentrations of MMPs (18-20). MMP-9, as one of the major MMPs, is present at high levels in malignant tumors and related to high numbers of distant metastases and poor prognosis $(21,22)$. However, the relationship between ROCK1 and MMP-9 in PTC has not been reported.

In the present study, we examined the ROCK1 expression in human PTC and investigated the biological role of ROCK1 in promoting the progression of PTC. The results showed that ROCK1 was overexpressed in both PTC tissues and cell lines, and was correlated inversely with the clinical outcomes. Furthermore, we found that ROCK1 promoted the xenograft tumor growth and the invasion of thyroid cancer cells through activating the expression of MMP-9. In addition, levels of MMP-9 positively correlated with the ROCK1 levels in PTC tissues. Taken together, our data suggest that ROCK1 might be a potential prognostic marker, and play an important role in the progression of PTC by upregulating MMP-9, which may be a potential therapeutic target for the treatment of PTC.

\section{Materials and methods}

Cell cultures and siRNA transfection. TPC-1 and K1 cell lines were obtained from the American Type Culture Collection (ATCC; Manassas, VA, USA) and maintained in 90\% RPMI1640 medium (Invitrogen, Carlsbad, CA, USA) supplementing with $10 \%$ fetal bovine serum (FBS) at $37^{\circ} \mathrm{C}$ with $5 \% \mathrm{CO}_{2}$ according to the standard protocols. The cells were transfected with Lipofectamine 2000 (Invitrogen) using siRNA (Shanghai GenePharma, Co., Ltd., Shanghai, China) according to the manufacturer's instructions. siRNA sequences of sense strands are as follows: siROCK1-1, 5'-GAAGCGAAUGACUUAUU ATT-3' and siROCK1-2, 5'-UAAGUAAGUCAUUCGCUUC TT-3'. In addition, MMP-9 inhibitor (Abcam, Cambridge, MA, USA) was used for invasion assays.

Patients and tissue specimens. Paraffin-embedded PTC specimens (=356) including 137 male patients and 219 female patients, who were histopathologically diagnosed at Sun Yat-sen Memorial Hospital of Sun Yat-sen University from January 2003 to July 2007, were evaluated in the present study. The male to female ratio was 1:1.60. Ages ranged from 16 to 79 years, and the median age was 43.7 years. Fifty-six patients $(15.73 \%)$ showed Hashimoto's thyroiditis (HT). The diameter of most tumors was $<4 \mathrm{~cm}$, the proportion of which was $70.51 \%$ $(153 / 217)$ in the weak ROCK1 expression group and $29.49 \%$
Table I. Clinicopathological features of patients with weak and strong ROCK1 expression in PTC.

\begin{tabular}{|c|c|c|c|c|c|}
\hline \multirow[b]{2}{*}{ Clinical features } & \multirow{2}{*}{$\begin{array}{l}\text { No. of } \\
\text { patients } \\
(356)\end{array}$} & \multicolumn{2}{|c|}{$\begin{array}{c}\text { ROCK1 } \\
\text { expression }\end{array}$} & \multirow[b]{2}{*}{$\chi^{2}$} & \multirow[b]{2}{*}{ P-value } \\
\hline & & $\begin{array}{l}\text { Weak } \\
(175)\end{array}$ & $\begin{array}{c}\text { Strong } \\
(181)\end{array}$ & & \\
\hline \multicolumn{6}{|l|}{ Age (years) } \\
\hline$<45$ & 194 & 101 & 93 & 1.439 & 0.2303 \\
\hline$\geq 45$ & 162 & 74 & 88 & & \\
\hline \multicolumn{6}{|l|}{ Sex } \\
\hline Male & 137 & 67 & 70 & 0.006 & 0.9400 \\
\hline Female & 219 & 108 & 111 & & \\
\hline \multicolumn{6}{|c|}{ Hashimoto's thyroiditis } \\
\hline No & 300 & 154 & 146 & 3.613 & 0.0573 \\
\hline Yes & 56 & 21 & 35 & & \\
\hline \multicolumn{6}{|l|}{ Tumor size (cm) } \\
\hline$\leq 4$ & 217 & 153 & 64 & 101.4 & $<0.0001^{\mathrm{a}}$ \\
\hline$\geq 4$ & 139 & 22 & 117 & & \\
\hline \multicolumn{6}{|c|}{ Lymphatic metastasis } \\
\hline No & 140 & 126 & 14 & 154.0 & $<0.0001^{\mathrm{a}}$ \\
\hline Yes & 216 & 49 & 167 & & \\
\hline \multicolumn{6}{|l|}{$\begin{array}{l}\text { Distant organ } \\
\text { metastasis }\end{array}$} \\
\hline No & 258 & 149 & 109 & 27.70 & $<0.0001^{\mathrm{a}}$ \\
\hline Yes & 98 & 26 & 72 & & \\
\hline \multicolumn{6}{|c|}{ Extrathyroid invasion } \\
\hline No & 176 & 126 & 50 & 70.09 & $<0.0001^{\mathrm{a}}$ \\
\hline Yes & 180 & 49 & 131 & & \\
\hline \multicolumn{6}{|l|}{ Vascular invasion } \\
\hline No & 252 & 147 & 105 & 29.06 & $<0.0001^{\mathrm{a}}$ \\
\hline Yes & 104 & 28 & 76 & & \\
\hline \multicolumn{6}{|l|}{ TNM stage } \\
\hline $\mathrm{I}+\mathrm{II}$ & 228 & 133 & 95 & 21.36 & $<0.0001^{\mathrm{a}}$ \\
\hline III+IV & 128 & 42 & 86 & & \\
\hline
\end{tabular}

PTC, papillary thyroid carcinoma; ROCK1, Rho-associated protein kinase 1; TNM, tumor-node-metastasis. ${ }^{*} \mathrm{P}<0.05$, statistical difference. $\mathrm{P}$-values were calculated by the $\chi^{2}$ test.

(64/217) in the strong ROCK1 expression group; 216 patients $(60.67 \%)$ suffered lymph node metastasis and 98 patients $(27.53 \%)$ had distant organ metastasis. Moreover, 50.56\% (180 cases) of the patients presented extrathyroid invasion, and $29.21 \%$ (104 cases) of the patients were found with vascular invasion, 228 patients (64.04\%) had stage I-II disease based on the tumor, node, metastasis (TNM) staging system (23), and the remaining 128 patients $(35.96 \%)$ had stage III-IV disease. The primary tumors of PTC and their corresponding adjacent non-cancerous tissues were collected for assays. Clinical information of the samples is presented in detail in the Table I. The study was performed in accordance with the policies of the Institutional Research Ethics Committee of Sun Yat-sen 
Memorial Hospital. Informed consents were obtained from the patients by BioBank, and the patients' personally identifiable information such as names, addresses and contact information were removed.

Immunohistochemistry. Immunohistochemistry (IHC) staining for ROCK1 was performed on 356 human PTC and the matched adjacent non-cancerous tissues. Briefly, paraffin-embedded sections ( $4 \mu \mathrm{m}$ thick) were deparaffinized, rehydrated, and microwave-heated for $15 \mathrm{~min}$ in $0.01 \mathrm{~mol} / 1$ citric buffer ( $\mathrm{pH}$ 6.0) for antigen retrieval. Then, $3 \%$ hydrogen peroxide was applied to block endogenous peroxidase activity. After 30 min of blocking with normal serum (Invitrogen), the primary rabbit anti-ROCK1 antibody (1:100 dilution; Abcam), or the corresponding control isotype $\mathrm{IgG}$ for antibody used were applied and incubated overnight at $4^{\circ} \mathrm{C}$. Slides were washed three times for $5 \mathrm{~min}$ each with phosphate-buffered saline (PBS). The biotinylated secondary antibody and the streptavidin-biotin complex were each incubated for $60 \mathrm{~min}$ at room temperature. After rinsing with PBS, the slides were immersed for $10 \mathrm{~min}$ in DAB (3,3-diaminobenzidine) (Sigma-Aldrich, St. Louis, MO, USA) solution $(0.4 \mathrm{mg} / \mathrm{ml}$, with $0.003 \%$ hydrogen peroxide), then monitored under a microscope. The reaction was terminated by immersing the slides in distilled water. Slides were then counterstained with hematoxylin, dehydrated and coverslipped.

Scoring of IHC staining. ROCK1-positive staining is localized in the cytoplasm of thyrocyte. The quantitative analysis of IHC staining analysis was performed for ROCK1 protein expression level in the tissue specimens. Two experienced investigators scored independently all the slides by the method previously described (24). Scores considered both the proportion of positive staining tumor cells and the staining intensity. The proportion of staining is graded as: 0 , no positive tumor cells; $1,<10 \%$ positive; $2,10-50 \%$ positive, and $3,>50 \%$ positive. The intensity of staining is determined as: 0 , no staining; 1, weak staining; 2, moderate staining and 3, strong staining. The staining index (SI) is calculated as the product of staining intensity and percentage of positive tumor cells, resulting in scores of 0, 1, 2, 3, 4, 6 and 9. Cut-off values for ROCK1 are chosen based on a measurement of heterogeneity using the log-rank test with respect to disease-free survival (DFS). We identified the optimal cut-off as: the SI score of $\geq 4$ was considered as strong ROCK1 expression, and $\leq 3$ as weak ROCK1 expression.

IHC staining for protein expression in tumor and adjacent non-cancerous tissues was quantitatively analyzed with the AxioVision Rel.4.6 computerized image analysis system assisted with the automatic measurement program (Carl Zeiss, Oberkochen, Germany). Briefly, to assess the mean optical density (MOD), which represents the strength of staining signals by measuring per positive pixels, we evaluated the stained sections at $\mathrm{x} 200$ magnification and ten representative staining fields of each section were analyzed.

RNA extraction and PCR assays. The fresh tissue specimens were collected with liquid nitrogen, and RNA was extracted using the TRIzol reagent and a reverse transcription kit (Invitrogen). The primers for quantitative real-time PCR assay were ROCK1-F, 5'-CAAATGAAGGTGAATGTAGAAA-3' and ROCK1-R: 5'-GCAGGAAAGTGGTAGAGTGT-3'; MMP-9-F, 5'-GCCTGGCACATAGTAGGCCC-3' and MMP-9-R, 5'-TCTCTCAGCCGGCATC-3'; GAPDH-F, 5'-GA CTCATGACCACAGTCCATGC-3' and GAPDH-R, 5'-AGA GGCAGGGATGATGTTCTG-3'. All primers were synthesized by Shanghai Generay Biotech, Co., Ltd, (Shanghai, China). The PCR reaction conditions were as follows: initial denaturation for $2 \mathrm{~min}$ at $94^{\circ} \mathrm{C}, 35$ cycles of denaturation for $30 \mathrm{sec}$, annealing for $30 \mathrm{sec}$ at $94^{\circ} \mathrm{C}$, and extension for $1 \mathrm{~min}$ at $72^{\circ} \mathrm{C}$, followed by $10 \mathrm{~min}$ at $72^{\circ} \mathrm{C}$. Expression data were normalized to the housekeeping gene GAPDH as a loading control.

Western blot analysis. Protein extracts were separated through $12 \%$ sodium dodecyl sulfate polyacrylamide gel electrophoresis, transferred to nitrocellulose membranes, probed with rabbit polyclonal antibodies against MMP-9 (1:1,000 dilution; Abcam), then incubated with peroxidase-conjugated goat antirabbit Ig secondary antibody (Oncogene Research Products, Cambridge, MA, USA) and visualized using chemiluminescence (Amersham, Arlington Heights, IL, USA).

Transduction with retroviral vectors. Ectopic expression of ROCK1 in thyroid cancer cells was achieved using retroviral vectors. Briefly, ROCK1 cDNA was cloned into retroviral transfer plasmid pMSCV to generate ROCK1 expression vector, co-transfecting in 293FT cells by using standard calcium phosphate transfection method as previously described (25). Thirty-six hours after the cotransfection, supernatants were collected and incubated with TPC-1 and K1 cells for $24 \mathrm{~h}$ for following assay.

Invasion assay. Cell invasion assays were performed in vitro as the method previously described (26). Transwell inserts for 24-well plates (Corning Costar, Cambridge, MA, USA) were coated with prediluted Matrigel (BD Biosciences, Bedford, MA, USA) and allowed to gel at $37^{\circ} \mathrm{C}$ for $30 \mathrm{~min}$. Cells were seeded at a density of $3 \times 10^{5}$ per insert and the lower chamber of the Transwell was filled with $500 \mu$ l Dulbecco's modified Eagle's medium (DMEM) supplemented with $10 \%$ fetal bovine serum (FBS). After $24 \mathrm{~h}$ of incubation, cells remaining on the upper surface of the Transwell membrane were removed by a cotton bud. Cells were quantified as the number of cells found in 6 random microscope fields in three independent inserts.

Tumor implantation. Thyroid cancer K1 cells were inoculated into the mammary fat pads of female nude mice. Mice were examined by palpation for tumor formation for more than 60 days. After tumors were detected, tumor size was measured every 7 days by calipers, and tumor volume was calculated as: Volume $\left(\mathrm{mm}^{3}\right)=$ Length $\times$ Width $^{2}$ x 0.5 every 7 days for 8 weeks. The animals were sacrificed when xenografts reached $\sim 1.5 \mathrm{~cm}$ in diameter and tumor engrafts were harvested, weighed and used for IHC staining. Tumor formation were determined by microscopic examination.

Statistical analysis. The statistical analyses were performed using the Statistical Package for Social Sciences software for Windows Version 13.0 (SPSS, Inc., Chicago, IL, USA). The 

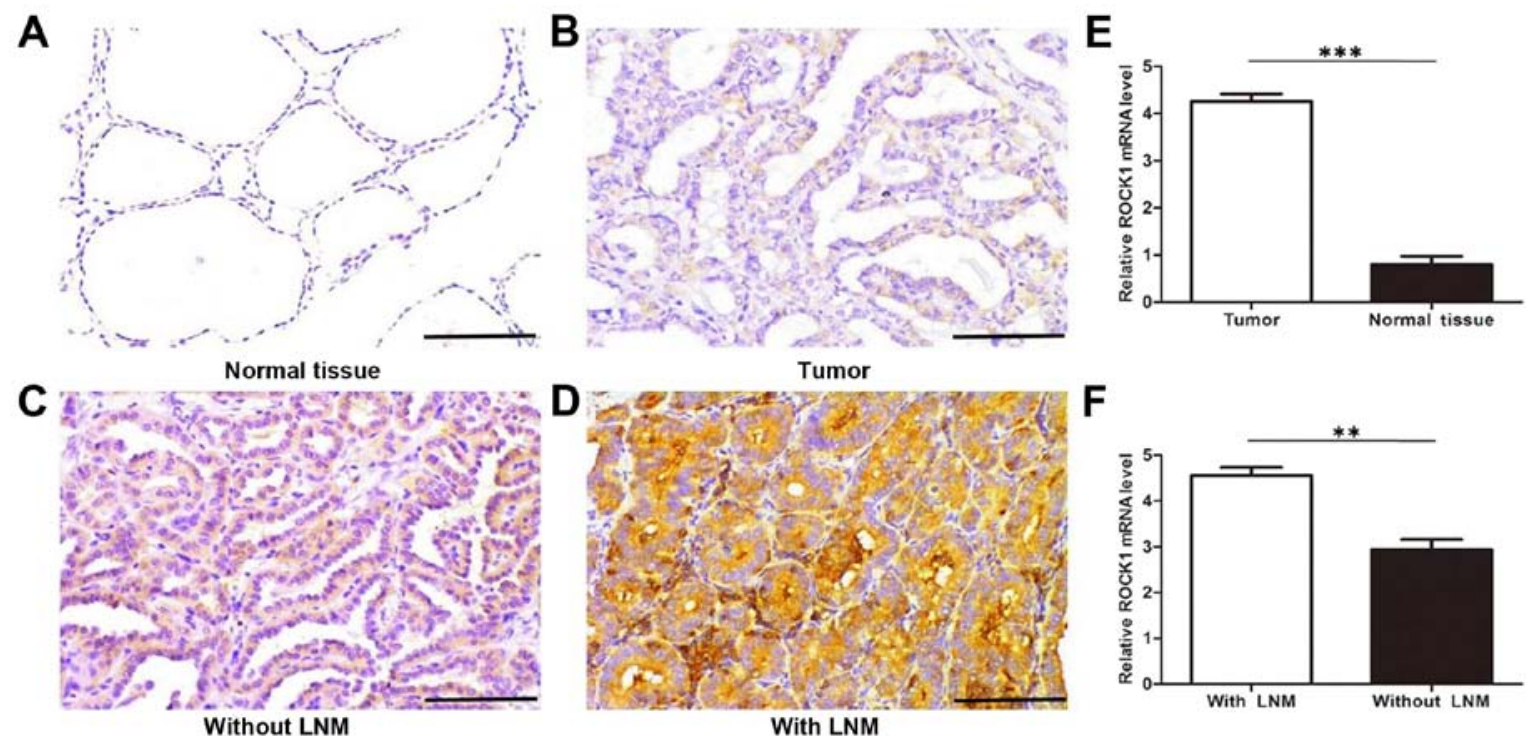

Figure 1. ROCK1 expression is correlated with PTC metastasis. (A and B) Representative images of immunohistochemistry (IHC) staining in formalin-fixed and paraffin-embedded normal adjacent tissues and papillary thyroid carcinoma tissues. (C and D) Representative images of IHC staining in PTC tissues without lymphatic metastasis and PTC with lymphatic metastasis. (E and F) ROCK1 mRNA expression was detected by qRT-PCR assay in PTC samples compared with normal adjacent samples, and PTC tissues with lymph node metastasis compared with PTC without. ${ }^{* *} \mathrm{P}<0.01,{ }^{* * *} \mathrm{P}<0.001$ from the Student's t-test; IHC, magnification, x200; scale bars, $100 \mu \mathrm{m}$. LNM, lymph node metastasis; IHC, immunohistochemistry; ROCK1, Rho-associated protein kinase 1.

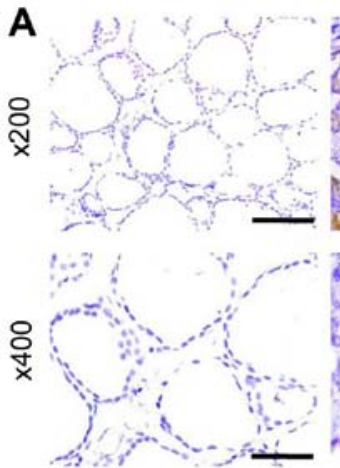

$\mathrm{N}$

B

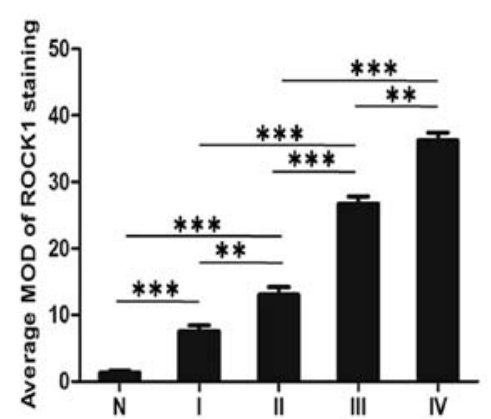

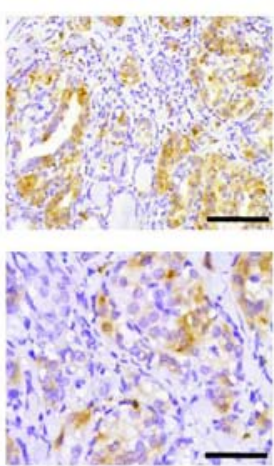

II

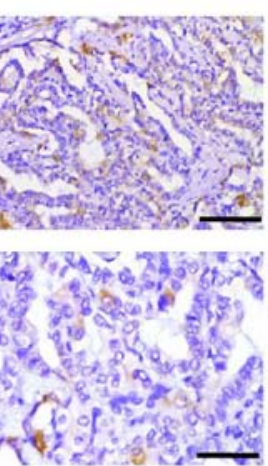

III

C

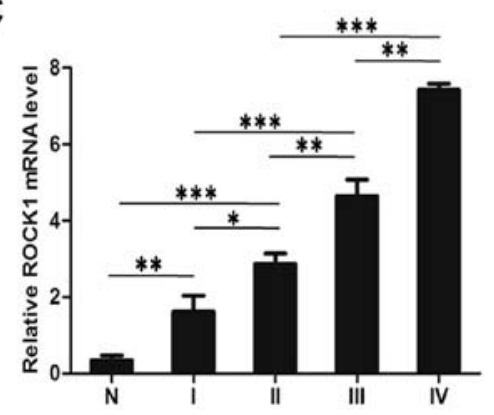

Figure 2. ROCK1 expression is associated with PTC stages. (A) Representative images from IHC assays of paraffin-embedded specimens of different stages of PTC tissue specimens and their adjacent non-cancerous tissues. Stage I ( $n=61)$, stage II $(n=167)$, stage III ( $n=88)$ and stage IV ( $n=40)$. (IHC, magnification, $\mathrm{x} 200$ and $\mathrm{x} 400$; scale bars, $100 \mu \mathrm{m}$ and $50 \mu \mathrm{m}$ ). (B) Comparative quantification of the MOD of ROCK1 staining among normal tissues and PTC specimens of different stages. (C) Verification of mRNA expression of ROCK1 in stages I-IV PTC tumors using qRT-PCR assay. ${ }^{*}<0.05{ }^{* * *} \mathrm{P}<0.01,{ }^{* * *} \mathrm{P}<0.001$ from the Student's t-test; IHC, immunohistochemistry; PTC, papillary thyroid carcinoma; ROCK1, Rho-associated protein kinase 1; MOD, mean optical density.

Chi-squared test was used to analyze the associations between ROCK1 expression and the clinicopathological features of PTC. Kaplan-Meier analysis was used for overall survival (OS), disease-free survival (DFS), lymph node recurrence-free survival (LNRFS) and distant recurrence-free survival (DRFS) calculations. OS was calculated as the time from the date of diagnosis to the date of death or the date of the last follow-up (if death did not occur). DFS was calculated as the time from the date of surgery to the date of the first recurrence or metastasis after surgery (in patients with recurrence or metastasis) or to the date of the last follow-up (in patients without recurrence and metastasis). LNRFS was defined as the time from the date 

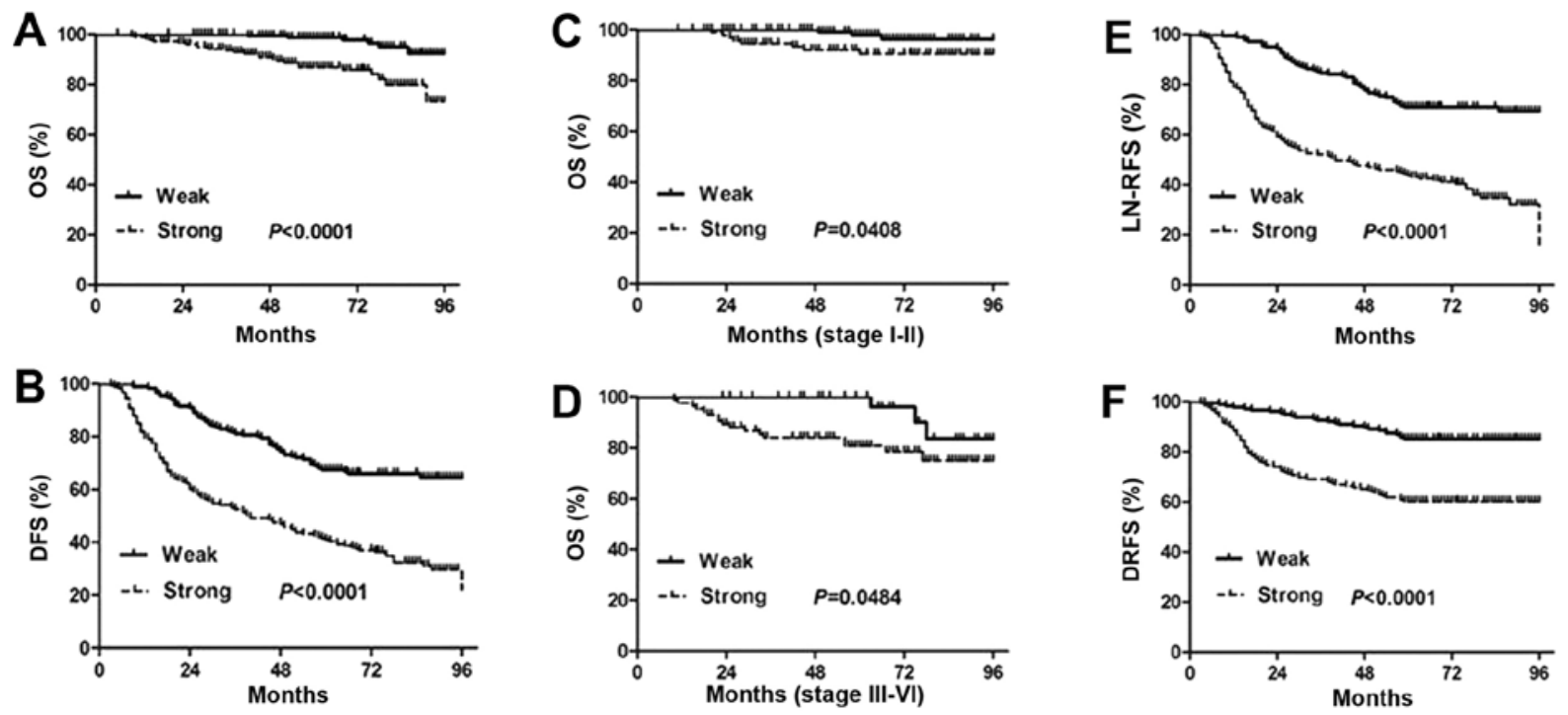

Figure 3. ROCK1 overexpression reduces patient's survival. (A and B) Strong ROCK1 expression was markedly associated with a reduced overall survival and disease-free survival ( $\mathrm{P}<0.0001)$. ( $\mathrm{C}$ and $\mathrm{D})$ Overall survival of PTC patients with strong ROCK1 expression was significantly decreased compared to those with weak expression in either stages $\mathrm{I}+\mathrm{II}$ subgroup $(\mathrm{P}=0.0408)$ or stages $\mathrm{III}+\mathrm{IV}$ subgroup $(\mathrm{P}=0.0484)$. (E and F) ROCK1 expression in PTC patients was significantly correlated with LN-RFS $(\mathrm{P}<0.0001)$ and DRFS $(\mathrm{P}<0.0001)$. P-values were calculated with the log-rank test. OS, overall survival; DFS, disease-free survival; LN-RFS, lymph node recurrence-free survival; DRFS, distant recurrence free survival; PTC, papillary thyroid carcinoma; ROCK1, Rho-associated protein kinase 1 .

of surgery to the date of lymph node relapse, and DRFS was defined as the time from the date of surgery to the date of distant recurrence. The prognostic significance of clinical and pathologic characteristics was determined using the univariate Cox regression analysis. Cox proportional hazards models were fitted for multivariate analysis. The correlation between ROCK1 and MMP-9 mRNA expression was determined using the Pearson's correlation test. Continuous data were compared using the Student's t-test. All statistical tests were two-tailed. Errors were SD of averaged results and P-values $<0.05$ were accepted as a significant difference.

\section{Results}

ROCK1 expression is correlated with PTC metastasis. Among 356 tumor samples, 50.84\% (181/356) of cases show strong ROCK1 expression, and 49.16\% (175/356) show weak expression (Table I). The diameter of most tumors is $<4 \mathrm{~cm}$, the proportion of which is $70.51 \%(153 / 217)$ in the weak ROCK 1 expression group and $29.49 \%$ (64/217) in the strong ROCK1 expression group. There is a significant association between strong ROCK1 expression and tumor size $(\mathrm{P}<0.0001)$, lymphatic metastasis $(\mathrm{P}<0.0001)$, distant organ metastasis $(\mathrm{P}<0.0001)$, extrathyroid invasion $(\mathrm{P}<0.0001)$, vascular invasion $(\mathrm{P}<0.0001)$ and $\mathrm{TNM}$ stage $(\mathrm{P}<0.0001)$. However, no association was found between the ROCK1 expression and other clinicopathological features. According to the data, ROCK1 expression in tumors might be useful for identifying the malignancy of PTC. Immunostaining results also show a significantly higher level of ROCK1 expression in PTC tissues compared with adjacent tissues (Fig. 1A and B). Moreover, these data further indicate that the ROCK1 protein expression is remarkably stronger in PTC with lymphatic metastasis than in those without lymphatic metastasis (Fig. 1C and D). To confirm these observations, we investigated the mRNA expression of ROCK1 in 125 cases of PTC tissues, and their normal adjacent tissues, 128 cases of PTC with lymph node metastasis, and 97 cases of PTC without lymph node metastasis using qRT-PCR analysis. The results indicate that ROCK1 mRNA expression is significantly higher in PTC tissues than normal adjacent tissues, and greatly higher in PTC tissues with lymph node metastasis than in those without metastasis (Fig. 1E and F). These findings suggest that elevated ROCK1 expression contributes to the invasiveness and metastasis of PTC.

ROCK1 expression is associated with PTC stages. The expression pattern of ROCK1 in 356 cases of paraffin-embedded PTC tissues includes 61 stage I, 167 stage II, 88 stage III, and 40 stage IV. As shown in Fig. 2A, ROCK1 expression is upregulated in all stages of PTC comparing with that in normal tissues. Notably, the ROCK1 expression is localized mainly in the cytoplasm of cancer cells. Moreover, comparative quantification of the MOD of ROCK1 staining among normal tissues and PTC specimens of different stages are summarized in Fig. 2B. The MOD of ROCK1 staining increases while PTC progresses from lower stages to higher $(\mathrm{P}<0.05)$. Furthermore, quantitative analysis verified that mRNA expression of ROCK1 in stages I-IV tumors was significantly higher than that in normal tissue, and also increases from lower stages to higher, by qRT-PCR assays $(\mathrm{P}<0.05$; Fig. $2 \mathrm{C})$. Taken as a whole, these data support the notion that the progression of PTC is associated with increased ROCK1 expression.

ROCK1 overexpression reduces patient's survival. KaplanMeier analysis using the log-rank test was performed to calculate the relationship between the ROCK1 expression and the survival rate in PTC patients. The results show that strong expression of ROCK1 is markedly associated with reduced overall survival and disease-free survival $(\mathrm{P}<0.0001$; Fig. 3A and B). The median survival time is significantly 
Table II. Univariate Cox regression analysis of disease-free survival in relation to clinicopathological features.

\begin{tabular}{|c|c|c|}
\hline \multirow[b]{2}{*}{ Clinical features } & \multicolumn{2}{|c|}{ PTC $(\mathrm{N}=356)$} \\
\hline & HR $(95 \% \mathrm{CI})$ & P-value \\
\hline Age (years) & & 0.457 \\
\hline$<45$ & 1 & \\
\hline$\geq 45$ & $0.463(0.125-2.835)$ & \\
\hline Tumor size $(\mathrm{cm})$ & & $0.028^{\mathrm{a}}$ \\
\hline$\leq 4$ & 1 & \\
\hline$>4$ & $2.605(1.764-3.879)$ & \\
\hline Lymphatic metastasis & & $<0.001^{\mathrm{a}}$ \\
\hline No & 1 & \\
\hline Yes & $6.223(0.834-9.431)$ & \\
\hline Distant organ metastasis & & $0.027^{\mathrm{a}}$ \\
\hline No & 1 & \\
\hline Yes & $2.856(1.210-12.081)$ & \\
\hline Extrathyroid invasion & & $0.003^{\mathrm{a}}$ \\
\hline No & 1 & \\
\hline Yes & $1.472(1.414-1.898)$ & \\
\hline Vascular invasion & & $<0.001^{\mathrm{a}}$ \\
\hline No & 1 & \\
\hline Yes & $2.235(1.114-11.797)$ & \\
\hline ROCK1 expression & & $<0.001^{\mathrm{a}}$ \\
\hline Weak & 1 & \\
\hline Strong & $4.541(1.349-8.782)$ & \\
\hline TNM stage & & $0.001^{\mathrm{a}}$ \\
\hline $\mathrm{I}+\mathrm{II}$ & 1 & \\
\hline III+IV & $4.893(1.806-13.785)$ & \\
\hline
\end{tabular}

PTC, papillary thyroid carcinoma; HR, hazard ratio; CI, confidence interval; ROCK1, Rho-associated protein kinase 1; TNM, tumor-nodemetastasis. ${ }^{*} \mathrm{P}<0.05$, statistically difference.

shorter in the patients with strong ROCK1 expression than those with weak ROCK1 expression. Moreover, the cumulative 8-year survival rate is only $86.18 \%(156 / 181)$ in the strong ROCK1 expression group, whereas it is $96.57 \%(169 / 175)$ in the weak ROCK1 expression group.

To further validate these findings, we next assessed the prognostic significance of ROCK1 expression in different subgroups of PTC patients stratified according to the TNM stage. Notably, strong ROCK1 expression is significantly correlated with shorter overall survival time in different PTC subgroups. Overall survival of patients with strong ROCK1 expression is significantly decreased compared to those with weak ROCK1 expression in either stages I+II subgroup $(\mathrm{N}=228 ; \mathrm{P}=0.0408$, log-rank; Fig. 3C) or stages III+IV subgroup ( $\mathrm{N}=128 ; \mathrm{P}=0.0484$, log-rank; Fig. 3D). Collectively, the data suggest that the level of ROCK1 expression strongly and significantly correlates with the prognosis of PTC and the disease outcome.
Table III. Multivariate Cox regression analysis of disease-free survival in relation to clinicopathological features.

\begin{tabular}{|c|c|c|}
\hline \multirow[b]{2}{*}{ Clinical features } & \multicolumn{2}{|c|}{ PTC (N=356) } \\
\hline & HR $(95 \% \mathrm{CI})$ & P-value \\
\hline Tumor size (cm) & & $0.032^{\mathrm{a}}$ \\
\hline$\leq 4$ & 1 & \\
\hline$>4$ & $1.275(1.021-1.592)$ & \\
\hline Lymphatic metastasis & & $<0.001^{\mathrm{a}}$ \\
\hline No & 1 & \\
\hline Yes & $2.380(1.361-4.142)$ & \\
\hline Distant organ metastasis & & $0.002^{\mathrm{a}}$ \\
\hline No & 1 & \\
\hline Yes & $4.284(1.697-10.931)$ & \\
\hline Extrathyroid invasion & & $<0.001^{\mathrm{a}}$ \\
\hline No & 1 & \\
\hline Yes & $1.557(1.229-2.204)$ & \\
\hline Vascular invasion & & $0.009^{\mathrm{a}}$ \\
\hline No & 1 & \\
\hline Yes & $2.237(1.209-4.516)$ & \\
\hline ROCK1 expression & & $<0.001^{\mathrm{a}}$ \\
\hline Weak & 1 & \\
\hline Strong & $2.895(1.697-5.126)$ & \\
\hline TNM stage & & $<0.001^{\mathrm{a}}$ \\
\hline $\mathrm{I}+\mathrm{II}$ & 1 & \\
\hline $\mathrm{III}+\mathrm{IV}$ & $1.553(1.275-1.903)$ & \\
\hline
\end{tabular}

PTC, papillary thyroid carcinoma; HR, hazard ratio; CI, confidence interval; ROCK1, Rho-associated protein kinase 1; TNM, tumor-nodemetastasis. ${ }^{~} \mathrm{P}<0.05$, statistically difference.

We also observed that ROCK1 expression in PTC patients is significantly correlated with LN-RFS $(\mathrm{P}<0.0001)$ and DRFS $(\mathrm{P}<0.0001$; Fig. 3E and F). Lymph node recurrence and distant metastasis are responsible for poor survival of PTC patients. PTC displays an indolent course and shows a 10-year survival rate of $\sim 90 \%$ (27). Our results may suggest a potential prognostic role of ROCK1 for PTC patients.

We next performed a univariate Cox regression analysis for disease-free survival (DFS). As shown in Table II, DFS is strongly correlated with ROCK1 expression $(\mathrm{P}<0.001)$ besides with tumor size $(\mathrm{P}=0.028)$, lymphatic metastasis $(\mathrm{P}<0.001)$, distant organ metastasis $(\mathrm{P}=0.027)$, extrathyroid invasion $(\mathrm{P}=0.003)$, vascular invasion $(\mathrm{P}<0.001)$ and $\mathrm{TNM}$ stage $(\mathrm{P}<0.001)$.

Furthermore, ROCK1 expression was also demonstrated as a useful prognostic biomarker for PTC patients by multivariate analysis (Table III) including tumor size (HR, 1.275; 95\% CI, 1.021-1.592; $\mathrm{P}=0.032$ ), lymphatic metastasis (HR, 2.380; 95\% CI, 1.361-4.142; $\mathrm{P}<0.001)$, distant organ metastasis (HR, 4.284; 95\% CI, 1.697-10.931; $\mathrm{P}=0.002)$, extrathyroid invasion $(\mathrm{HR}$, 1.557; 95\% CI, 1.229-2.204; P<0.001), vascular invasion (HR, 

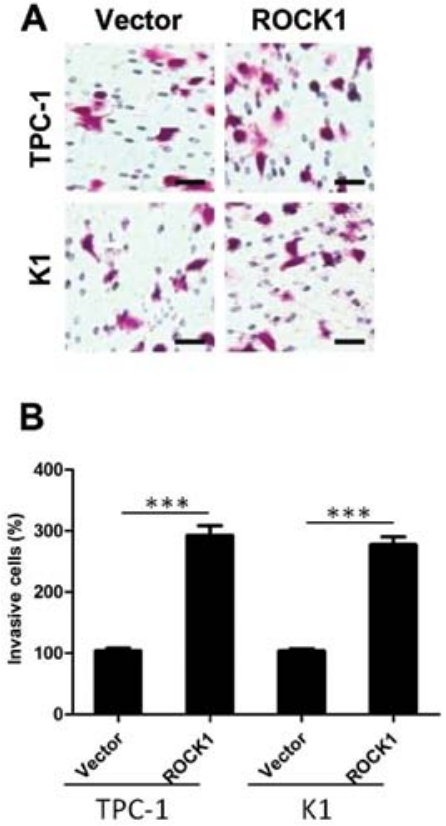

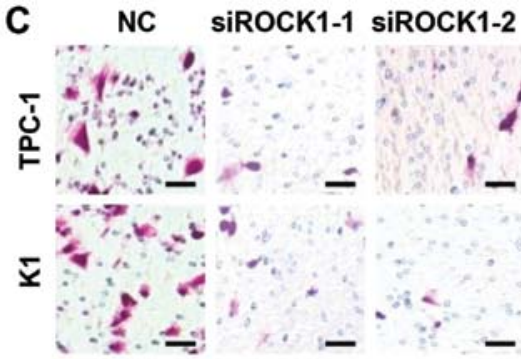

D

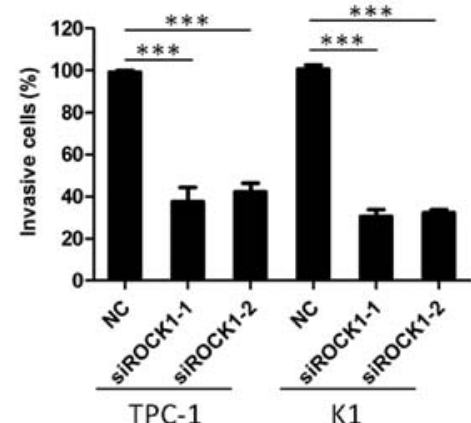

Figure 4. ROCK1 promotes invasiveness on PTC cell lines. (A and B) ROCK1 overexpression efficiently promoted TPC-1 and K1 thyroid cancer cell invasion as demonstrated by using the invasion assay. (C and D) Knockdown of ROCK1 expression by specific siRNA markedly inhibited the invasive capability of TPC-1 and K1 thyroid cancer cells. ${ }^{* * *} \mathrm{P}<0.001$ from the Student's t-test; scale bars, $20 \mu \mathrm{m}$. ROCK1, Rho-associated protein kinase 1; PTC, papillary thyroid carcinoma.
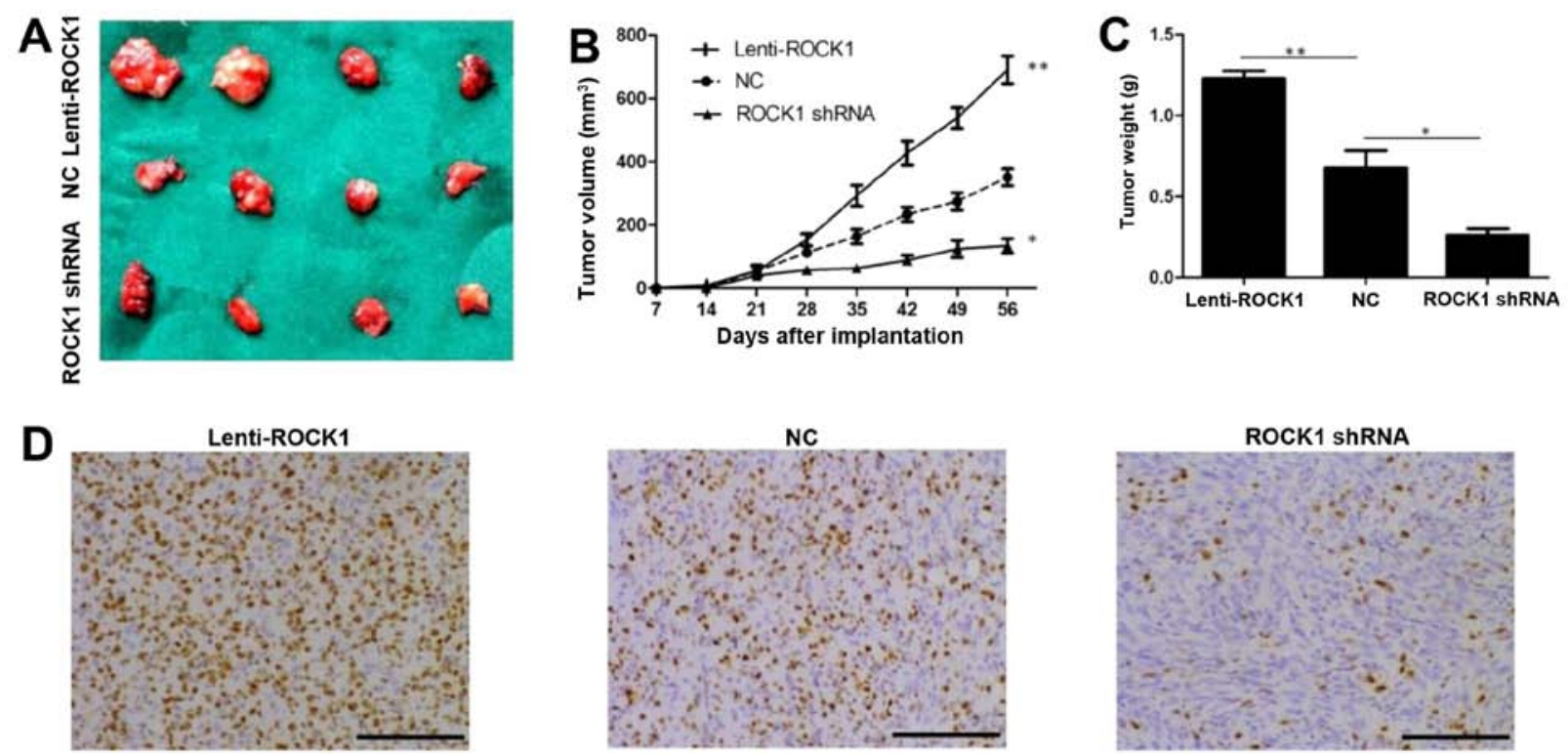

Figure 5. ROCK1 promotes thyroid cancer K1 cells growth in vivo. The nude mice were subcutaneously injected with $1 \times 10^{6}$ thyroid cancer K1 cells with lenti-ROCK1, ROCK1 shRNA or negative control, respectively. (A) Representative images of the tumors on week 8 are shown. (B) Xenograft assay revealed that knockdown of ROCK1 decreased the volume of the xenograft tumors, while overexpression of ROCK1 showed a proliferative tendency with significantly greater tumor volume ( $\left.{ }^{*} \mathrm{P}<0.05,{ }^{* *} \mathrm{P}<0.01\right)$. (C) Knockdown of ROCK1 decreased the weight of the xenograft tumors, while overexpression of ROCK1 showed a proliferative tendency with significantly greater tumor weight $\left({ }^{*} \mathrm{P}<0.05,{ }^{* *} \mathrm{P}<0.01\right)$. (D) Representative images of immunohistochemical detection of Ki-67 staining in K1 xenograft tumors from control, lenti-ROCK1 or ROCK1 shRNA-treated mice, respectively (IHC, magnification, x200; scale bars, $100 \mu \mathrm{m}$ ). ROCK1, Rho-associated protein kinase 1; PTC, papillary thyroid carcinoma; MMP-9, matrix metalloproteinase-9.

$2.237 ; 95 \%$ CI, 1.209-4.516; P=0.009), TNM stage (HR, 1.553; 95\% CI, 1.275-1.903; $\mathrm{P}<0.001)$. Collectively, these data suggest that ROCK1 might serve as a previously unappreciated prognostic predictor of the long-term survival of PTC patients.

ROCK1 promotes invasiveness on PTC cell lines. To investigate whether and how ROCK1 impacts on the aggressive nature of PTC cells, TPC-1 and K1 thyroid cancer cells were engineered to overexpress ROCK1 and tested for their ability of invasion. As shown in Fig. 4A and B, the invasion assay demonstrated that ROCK1 overexpression efficiently promoted PTC cells invasion $(\mathrm{P}<0.001)$. To further confirm the role of ROCK1 in cancer cell invasion, we knocked down ROCK1 expression by specific siRNA, and the invasive capability of 
A

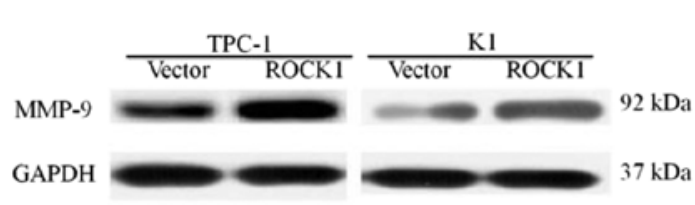

B
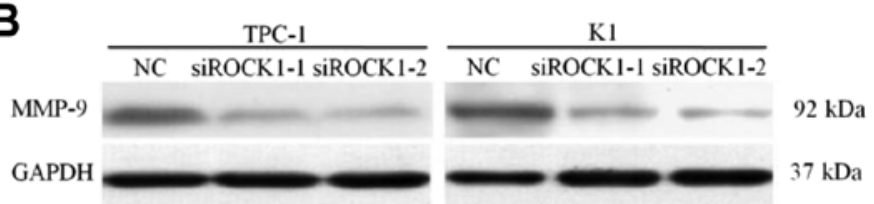

C

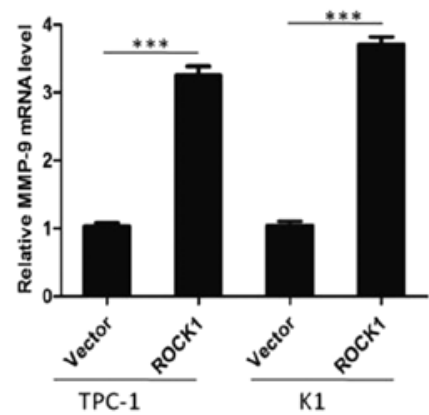

$\mathbf{F}$

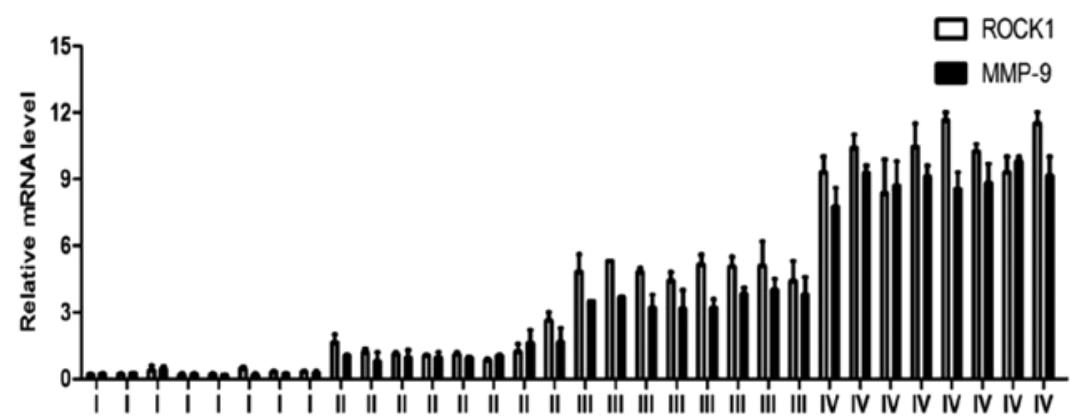

E
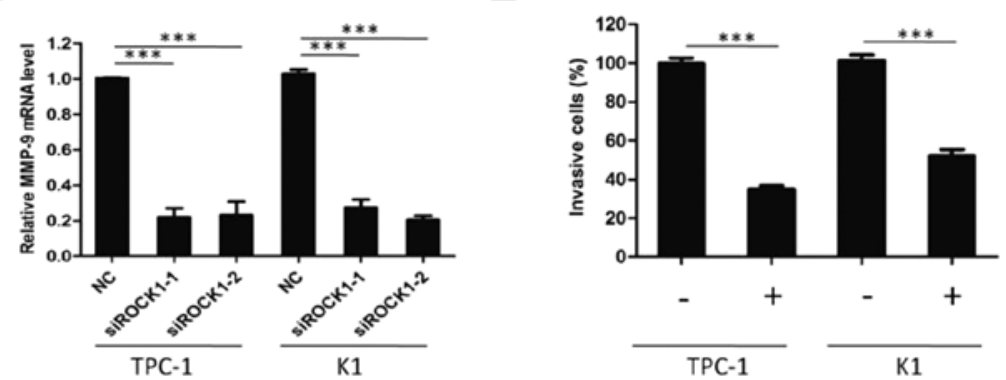

G

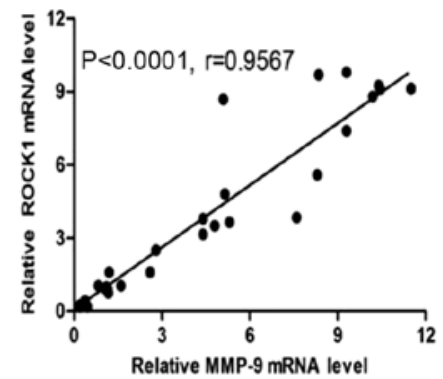

Figure 6. ROCK1 may promote PTC invasion through MMP-9. (A and C) ROCK1 overexpression significantly increased the expression of MMP-9 by using western blot analysis and qRT-PCR assay $\left({ }^{* * *} \mathrm{P}<0.001\right)$. (B and D) Knockdown of ROCK1 expression markedly decreased the protein and mRNA level of MMP-9 by using western blot analysis and qRT-PCR assay $\left({ }^{* * *} \mathrm{P}<0.001\right)$. (E) Invasion assays were performed in the presence, or absence of the MMP-9 inhibitor $(30 \mu \mathrm{mol} / \mathrm{l})\left({ }^{* * *} \mathrm{P}<0.001\right)$. (F) ROCK1 and MMP-9 mRNA expression level studied by qRT-PCR increased from stages I to IV with similar patterns in PTC tissues. Stage I $(n=8)$, stage II $(n=8)$, stage III $(n=8)$ and stage IV $(n=8)$. (G) Levels of MMP-9 positively correlated with the ROCK1 levels in PTC tissues $(\mathrm{n}=32, \mathrm{r}=0.9567, \mathrm{P}<0.0001)$.

TPC-1 and K1 thyroid cancer cells was markedly inhibited $(\mathrm{P}<0.001)$ (Fig. 4C and D), suggesting that ROCK1 plays a role in PTC invasion.

ROCK1 promotes tumor growth in vivo. To determine if ROCK1 is involved in tumor growth in vivo, we used tumor implantation model to assess tumor growth using thyroid cancer K1 cells. The nude mice were subcutaneously injected with $1 \times 10^{6}$ thyroid cancer K1 cells with lenti-ROCK1, ROCK1 shRNA or negative control, respectively. Representative images of the xenograft tumors on week 8 are shown in Fig. 5A. Our data revealed that knockdown of ROCK1 decreased the volume of the xenograft tumors, while overexpression of ROCK1 showed a proliferative tendency with significantly greater tumor volume $(\mathrm{P}<0.05$; Fig. $5 \mathrm{~B})$. We also observed similar results on tumor weight in mice injected with thyroid cancer K1 cells with lenti-ROCK1 and ROCK1 shRNA compared with control mice ( $\mathrm{P}<0.05$; Fig. 5C). Noteworthy, our data indicated that IHC detection of Ki-67 staining in $\mathrm{K} 1$ xenograft tumors showed similar patterns (Fig. 5D). Together, these data suggest that inhibiting ROCK1 activity might be an effective approach for treating PTC.

ROCK1 may promote PTC invasion through MMP-9. To understand the mechanism by which ROCK1 enhances cancer cell invasion, we analyzed MMP-9 expression in ROCK1overexpressed cells by in vitro assays. ROCK1 overexpression significantly increased the expression of MMP-9 protein (Fig. 6A), indicating a possible correlation between MMP-9 and ROCK1. Moreover, qRT-PCR showed that ectopic expression of ROCK1 resulted in MMP-9 mRNA overexpression (Fig. 6C). In contrast, the knockdown of ROCK1 expression markedly decreased both protein and mRNA level of MMP-9 (Fig. 6B and D). When MMP-9 expression was suppressed by a specific inhibitor, the effect of ROCK1 in enhancing PTC cell invasion was blocked as shown in Fig. 6E, suggesting that MMP-9 is necessary for ROCK1 promoting PTC cell invasion.

In agreement, ROCK1 and MMP-9 mRNA expression level studied by qRT-PCR increased from stages I-IV with similar patterns in PTC tissues (Fig. 6F). Levels of MMP-9 positively correlated with the ROCK1 levels in PTC tissues (Fig. 6G; $\mathrm{r}=0.9567, \mathrm{P}<0.0001)$. Collectively, our results confirm that ROCK1 promotes cell invasion in PTC through the upregulation of MMP-9.

\section{Discussion}

To the best of our knowledge, for the first time, our results confirmed that ROCK1 is overexpressed in PTC, and that the ROCK1 overexpression is significantly associated with the 
clinical and pathological features, as well as the prognosis, of PTC. Consistent with this, we have demonstrated that ROCK1 might promote the invasiveness of cancer cells, possibly through upregulating MMP-9. This study also demonstrated the role of ROCK1 in invasion malignant tumors. Moreover, ROCK1 may be a powerful predictor of the outcome of papillary thyroid cancer patients.

ROCK1 enhanced the invasion of cancer cells and direct phosphorylation of myosin light chain, which leads to increased cell migration and invasion (28-30). ROCK1 overexpression has been reported in several cancer types. Based on their oncogenic activity, ROCK1 was examined as therapeutic targets in various tumors, such as lung tumors $(13,31)$, glioblastoma (32), osteosarcoma $(33,34)$, prostate $(35,36)$, breast $(37,38)$, ovarian cancer (39), hepatocellular carcinoma (40) and bladder cancer (41). Moreover, ROCK1 was indicated as an independent predictor of patients survival.

However, the influence of ROCK1 in PTC has remained unknown. Evidence in support of a connection such as provided by the present study, includes positive results of ROCK1 detection in tumor tissues paired with non-tumorous tissue, and in a cohort of 356 PTC specimens and two PTC cell lines. Further support for a possible role of ROCK1 in PTC pathogenesis derived from the analysis that revealed a strong correlation of ROCK1 expression with the IHC staging and inversely, with the survival of the disease. Moreover, our results suggest that a strong ROCK1 expression might be associated with tumor size, lymphatic metastasis, distant organ metastasis, extrathyroid invasion, vascular invasion and TNM stage, further implying an involvement of ROCK1 in the incidence and development of PTC. In addition, we demonstrated an association between strong ROCK1 expression and the prognosis of patients with PTC using Kaplan-Meier survival curves. Our findings indicate that a strong expression of ROCK1 indicates worse OS, DFS, LN-RFS and DRFS. At the same time, our univariate and multivariate analysis using Cox proportional hazards regression model showed that strong expression of ROCK1 was related to PTC patient prognosis. Knockdown of ROCK1 decreased the volume and weight of the xenograft tumors, while overexpression of ROCK1 showed a proliferative tendency with significantly greater tumor volume and weight in vivo. Based on these results, our data not only suggest that ROCK1 is likely to be biologically involved in the progression of PTC, but also might represent a valuable independent prognostic biomarker for the disease.

While this study has provided strong evidence for the upregulation of ROCK1 expression in PTC, the molecular mechanism underlying the observed ROCK1 upregulation is yet to be elucidated. Multiple steps are involved in the invasion of PTC cells, including cancer cell attachment to ECM, degradation of ECM components and subsequent infiltration into adjacent normal tissue (42). The accomplishment of this process, as shown by several lines of research, is largely attributable to the activation of MMPs. Among the MMPs, a subset called gelatinases, consisting of MMP-2 and MMP-9, has gained the most attention in studies on the acquisition of invasive and metastatic tumor properties, as they degrade collagen IV, the major component of the basement membrane $(18,43)$.

Furthermore, MMP-9 is of special interest since its basal expression is normally low, whereas it is highly expressed in most human cancers in response to various growth factors and cytokines $(43,44)$. It has been shown that MMP-9-deficient exhibit impaired metastasis formation and tumor growth. In this respect, upregulation of MMP-9 expression in various types of human cancers contributes to tumor progression, invasion and metastasis $(18,45,46)$. In addition, there are more evidence demonstrating that MMP-9 was overexpressed in various types of tumors when compared to normal tissue, including in papillary thyroid cancer $(47,48)$. On the other hand, emerging evidence indicate that the upregulation of ROCK1 protein enhanced invasion of cells of various types of tumors. To the best of our knowledge, however, the connection of ROCK1 and MMP-9 and their impact on the outcome of PTC patients have not been studied. This study first demonstrates the pathological role of ROCK1 in enhancing MMP-9 expression and mediating the invasive phenotype of thyroid cancer cells. Moreover, levels of MMP-9 positively correlated with the ROCK1 levels in PTC tissues.

In conclusion, to the best of our knowledge, this is the first report on the relationship between ROCK1 and prognosis in patients with PTC. This study indicates that ROCK1 is an independent prognostic marker for human PTC and that its strong expression contributes to papillary thyroid carcinoma progression by enhancing MMP-9 expression and tumor invasion. These results, together with the correlation between ROCK1/MMP-9 pathway and metastasis in papillary thyroid carcinoma patients, point towards the importance of targeting ROCK1 as a novel approach for the treatment of papillary thyroid carcinoma.

\section{Acknowledgements}

The present study was supported by grants from the National Natural Science Foundation of China (81702654), the Natural Science Foundation of Guangdong Province (2017A030313642), the grant (2013) 163 from the Key Laboratory of Malignant Tumor Molecular Mechanism and Translational Medicine of Guangzhou Bureau of Science and Information Technology and the grant K1809001 from the Key Laboratory of Malignant Tumor Gene Regulation and Target Therapy of Guangdong Higher Education Institutes.

\section{References}

1. Zeiger MA and Schneider EB: BRAF V600E mutation independently predicts central compartment lymph node metastasis in patients with papillary thyroid cancer. Ann Surg Oncol 20: 3-4, 2013.

2. Zivaljevic V, Slijepcevic N, Sipetic S, Paunovic I, Diklic A, Zoric G and Kalezic N: Risk factors for well-differentiated thyroid cancer in men. Tumori 99: 458-462, 2013.

3. Sipos JA and Mazzaferri EL: Thyroid cancer epidemiology and prognostic variables. Clin Oncol (R Coll Radiol) 22: 395-404, 2010.

4. Li Z, Huang X, Xu J, Su Q, Zhao J and Ma J: miR-449 overexpression inhibits papillary thyroid carcinoma cell growth by targeting RET kinase- $\beta$-catenin signaling pathway. Int $\mathrm{J}$ Oncol 49: 1629-1637, 2016.

5. Cheng S, Serra S, Mercado M, Ezzat S and Asa SL: A highthroughput proteomic approach provides distinct signatures for thyroid cancer behavior. Clin Cancer Res 17: 2385-2394, 2011.

6. Agrawal N, Akbani R, Aksoy BA, Ally A, Arachchi H, Asa SL, Auman JT, Balasundaram M, Balu S, Baylin SB, et al; Cancer Genome Atlas Research Network: Integrated genomic characterization of papillary thyroid carcinoma. Cell 159: 676-690, 2014. 
7. Beninato T, Scognamiglio T, Kleiman DA, Uccelli A, Vaca D, Fahey TJ III and Zarnegar R: Ten percent tall cells confer the aggressive features of the tall cell variant of papillary thyroid carcinoma. Surgery 154: 1331-1336, discussion 1336, 2013.

8. Lee CW, Roh JL, Gong G, Cho KJ, Choi SH, Nam SY and Kim SY: Risk factors for recurrence of papillary thy roid carcinoma with clinically node-positive lateral neck. Ann Surg Oncol 22: 117-124, 2015

9. Zhu J, Wang X, Zhang X, Li P and Hou H: Clinicopathological features of recurrent papillary thyroid cancer. Diagn Pathol 10 96, 2015.

10. Saito K, Ozawa Y, Hibino K and Ohta Y: FilGAP, a Rho/ Rho-associated protein kinase-regulated GTPase-activating protein for Rac, controls tumor cell migration. Mol Biol Cell 23: 4739-4750, 2012.

11. Banyard J, Anand-Apte B, Symons M and Zetter BR: Motility and invasion are differentially modulated by Rho family GTPases. Oncogene 19: 580-591, 2000.

12. Morgan-Fisher M, Wewer UM and Yoneda A: Regulation of ROCK activity in cancer. J Histochem Cytochem 61: 185-198, 2013.

13. Vigil D, Kim TY, Plachco A, Garton AJ, Castaldo L, Pachter JA, Dong H, Chen X, Tokar B, Campbell SL, et al: ROCK1 and ROCK2 are required for non-small cell lung cancer anchorageindependent growth and invasion. Cancer Res 72: 5338-5347, 2012.

14. Guan R, Xu X, Chen M, Hu H, Ge H, Wen S, Zhou S and Pi R Advances in the studies of roles of Rho/Rho-kinase in diseases and the development of its inhibitors. Eur J Med Chem 70: 613-622, 2013

15. Azab AK, Kleinstern J, Doviner V, Orkin B, Srebnik M, Nissan A and Rubinstein A: Prevention of tumor recurrence and distant metastasis formation in a breast cancer mouse model by biodegradable implant of 131I-norcholesterol. J Control Release 123 $116-122,2007$

16. Liu FH, Kuo SF, Hsueh C, Chao TC and Lin JD: Postoperative recurrence of papillary thyroid carcinoma with lymph node metastasis. J Surg Oncol 112: 149-154, 2015.

17. Werb Z: ECM and cell surface proteolysis: Regulating cellular ecology. Cell 91: 439-442, 1997.

18. Kessenbrock K, Plaks V and Werb Z: Matrix metalloproteinases: Regulators of the tumor microenvironment. Cell 141: 52-67, 2010.

19. Deryugina EI and Quigley JP: Matrix metalloproteinases and tumor metastasis. Cancer Metastasis Rev 25: 9-34, 2006.

20. Bottino J, Gelaleti GB, Maschio LB, Jardim-Perassi BV and de Campos Zuccari DA: Immunoexpression of ROCK-1 and MMP-9 as prognostic markers in breast cancer. Acta Histochem 116: 1367-1373, 2014.

21. Wang JR, Gan WJ, Li XM, Zhao YY, Li Y, Lu XX, Li JM and Wu H: Orphan nuclear receptor Nur77 promotes colorectal cancer invasion and metastasis by regulating MMP-9 and E-cadherin. Carcinogenesis 35: 2474-2484, 2014.

22. Gao J,Liu X, Yang F, Liu T, Yan Q and Yang X: By inhibiting Ras/ Raf/ERK and MMP-9, knockdown of EpCAM inhibits breast cancer cell growth and metastasis. Oncotarget 6: 27187-27198, 2015.

23. Haugen BR, Alexander EK, Bible KC, Doherty GM, Mandel SJ, Nikiforov YE, Pacini F, Randolph GW, Sawka AM, Schlumberger M, et al: 2015 American Thyroid Association Management Guidelines for Adult Patients with Thyroid Nodules and Differentiated Thyroid Cancer: The American Thyroid Association Guidelines Task Force on Thyroid Nodules and Differentiated Thyroid Cancer. Thyroid 26: 1-133, 2016

24. Li J, Guan HY, Gong LY, Song LB, Zhang N, Wu J, Yuan J, Zheng YJ, Huang ZS and Li M: Clinical significance of sphingosine kinase-1 expression in human astrocytomas progression and overall patient survival. Clin Cancer Res 14: 6996-7003, 2008.

25. Gong C, Qu S, Liu B, Pan S, Jiao Y, Nie Y, Su F, Liu Q and Song E: MiR-106b expression determines the proliferation paradox of TGF- $\beta$ in breast cancer cells. Oncogene 34: 84-93, 2015.

26. Chen J, Yao Y, Gong C, Yu F, Su S, Chen J, Liu B, Deng H, Wang F, Lin L, et al: CCL18 from tumor-associated macrophages promotes breast cancer metastasis via PITPNM3. Cancer Cell 19: 541-555, 2011.

27. Akaishi J, Sugino K, Kameyama K, Masaki C, Matsuzu K, Suzuki A, Uruno T, Ohkuwa K, Shibuya H, Kitagawa W, et al: Clinicopathologic features and outcomes in patients with diffuse sclerosing variant of papillary thyroid carcinoma. World J Surg 39: 1728-1735, 2015 .
28. Lochhead PA, Wickman G, Mezna M and Olson MF: Activating ROCK1 somatic mutations in human cancer. Oncogene 29: 2591-2598, 2010

29. Pinner S and Sahai E: PDK1 regulates cancer cell motility by antagonising inhibition of ROCK1 by RhoE. Nat Cell Biol 10: 127-137, 2008.

30. Liu X, Choy E, Hornicek FJ, Yang S, Yang C, Harmon D, Mankin $\mathrm{H}$ and Duan Z: ROCK1 as a potential therapeutic target in osteosarcoma. J Orthop Res 29: 1259-1266, 2011.

31. Li J, Song Y, Wang Y, Luo J and Yu W: MicroRNA-148a suppresses epithelial-to-mesenchymal transition by targeting ROCK1 in non-small cell lung cancer cells. Mol Cell Biochem 380: 277-282, 2013.

32. Mertsch S, Oellers P, Wendling M, Stracke W and Thanos S: Dissecting the inter-substrate navigation of migrating glioblastoma cells with the stripe assay reveals a causative role of ROCK Mol Neurobiol 48: 169-179, 2013.

33. Wang W, Zhou X and Wei M: MicroRNA-144 suppresses osteosarcoma growth and metastasis by targeting ROCK 1 and ROCK2. Oncotarget 6: 10297-10308, 2015.

34. Cai H, Lin L, Cai H, Tang M and Wang Z: Combined microRNA340 and ROCK1 mRNA profiling predicts tumor progression and prognosis in pediatric osteosarcoma. Int J Mol Sci 15: 560-573, 2014.

35. Xu B, Huang Y, Niu X, Tao T, Jiang L, Tong N, Chen S, Liu N, Zhu $\mathrm{W}$ and Chen M: Hsa-miR-146a-5p modulates androgenindependent prostate cancer cells apoptosis by targeting ROCK1. Prostate 75: 1896-1903, 2015.

36. Kroiss A, Vincent S, Decaussin-Petrucci M, Meugnier E, Viallet J, Ruffion A, Chalmel F, Samarut J and Allioli N: Androgen-regulated microRNA-135a decreases prostate cancer cell migration and invasion through downregulating ROCK1 and ROCK2. Oncogene 34: 2846-2855, 2015.

37. Raviraj V, Fok S, Zhao J, Chien HY, Lyons JG, Thompson EW and Soon L: Regulation of ROCK1 via Notch1 during breast cancer cell migration into dense matrices. BMC Cell Biol 13: 12, 2012.

38. Gilkes DM, Xiang L, Lee SJ, Chaturvedi P, Hubbi ME, Wirtz D and Semenza GL: Hypoxia-inducible factors mediate coordinated RhoA-ROCK1 expression and signaling in breast cancer cells. Proc Natl Acad Sci USA 111: E384-E393, 2014.

39. Dai W, Teodoridis JM, Zeller C, Graham J, Hersey J, Flanagan JM, Stronach E, Millan DW, Siddiqui N, Paul J, et al: Systematic $\mathrm{CpG}$ islands methylation profiling of genes in the wnt pathway in epithelial ovarian cancer identifies biomarkers of progression-free survival. Clin Cancer Res 17: 4052-4062, 2011.

40. Liu H, Li W, Chen C, Pei Y and Long X: MiR-335 acts as a potential tumor suppressor miRNA via downregulating ROCK1 expression in hepatocellular carcinoma. Tumour Biol 36: 6313-6319, 2015

41. Majid S, Dar AA, Saini S, Shahryari V, Arora S, Zaman MS, Chang I, Yamamura S, Chiyomaru T, Fukuhara S, et al: MicroRNA-1280 inhibits invasion and metastasis by targeting ROCK1 in bladder cancer. PLoS One 7: e46743, 2012.

42. Kummer NT, Nowicki TS, Azzi JP, Reyes I, Iacob C, Xie S, Swati I, Darzynkiewicz Z, Gotlinger KH, Suslina N, et al: Arachidonate 5 lipoxygenase expression in papillary thyroid carcinoma promotes invasion via MMP-9 induction. J Cell Biochem 113: 1998-2008, 2012.

43. Egeblad M and Werb Z: New functions for the matrix metalloproteinases in cancer progression. Nat Rev Cancer 2: 161-174, 2002.

44. Brown-Clay JD, Shenoy DN, Timofeeva O, Kallakury BV, Nandi AK and Banerjee PP: PBK/TOPK enhances aggressive phenotype in prostate cancer via $\beta$-catenin-TCF/LEF-mediated matrix metalloproteinases production and invasion. Oncotarget 6 : 15594-15609, 2015.

45. Wille C, Köhler C, Armacki M, Jamali A, Gössele U, Pfizenmaier K, Seufferlein T and Eiseler T: Protein kinase D2 induces invasion of pancreatic cancer cells by regulating matrix metalloproteinases. Mol Biol Cell 25: 324-336, 2014.

46. Ai F, Zhang X, Li X, Qin Z, Ye Q, Tian L, Tang A, Li N, Li G, Ma J, et al: Up-regulation of matrix metalloproteinases in a mouse model of chemically induced colitis-associated cancer: The role of microRNAs. Oncotarget 6: 5412-5425, 2015.

47. Decock J, Thirkettle S, Wagstaff L and Edwards DR: Matrix metalloproteinases: Protective roles in cancer. J Cell Mol Med 15: 1254-1265, 2011.

48. Hadler-Olsen E, Winberg JO and Uhlin-Hansen L: Matrix metalloproteinases in cancer: Their value as diagnostic and prognostic markers and therapeutic targets. Tumour Biol 34: 2041-2051, 2013. 